\title{
FP-LMTO Investigation of the Structural and Electronic Properties of $\mathrm{Cu}_{x} \mathrm{Ag}_{1-x}$ I Alloys
}

\author{
Mohammed Ameri ${ }^{*}$, Noureddine Bouzouira ${ }^{1}$, Mohammed Doui-Aici ${ }^{2}$, Rabah Khenata ${ }^{3,4}$, \\ Abdelkader Yakoubi ${ }^{5}$, Boualem Abidri ${ }^{1}$, Nouredinne Moulay ${ }^{1}$, Mohammed Maachou ${ }^{1}$
}

${ }^{1}$ Département de Physique, Faculté des Sciences, Université Djillali Liabès, Sidi-Bel-Abbés, Algérie; ${ }^{2}$ Laboratoire des Matériaux Appliqués, Centre de Recherche (Ex:CFTE), Route de Mascara, Université de Sidi-Bel-Abbès, Sidi-Bel-Abbès, Algérie; ${ }^{3}$ Laboratoire de Physique Quantique et de Modélisation Mathématique de la Matière (LPQ3 M), Université de Mascara, Mascara, Algérie; ${ }^{4}$ Department of Physics and Astronomy, Faculty of Science, King Saud University, Riyadh, Saudi Arabia; ${ }^{5}$ Laboratoire Modeling and Simulation Materials Sciences Laboratory, Sidi-Bel-Abbès University, Sidi-Bel-Abbès,Algeria.

Email: 1ttnsameri@yahoo.fr

Received December $28^{\text {th }}, 2010$; revised March 28 ${ }^{\text {th }}, 2011$; accepted May $8^{\text {th }}, 2011$.

\begin{abstract}
The structural and electronic properties of the ternary $\mathrm{Cu}_{x} \mathrm{Ag}{ }_{1-x} I$, alloy have been calculated, using the full-potential linear muffin-tin-orbital (FP-LMTO) method based on density functional theory, within both the local density approximation and the generalized gradient approximation (GGA). The equilibrium lattice constants and the bulk modulus are compared with previous theoretical calculations. The concentration dependence of the electronic band structure and the direct-indirect band gaps is also investigated. Using the approach of Zunger and co-workers the microscopic origins of the gap bowing were also explained.
\end{abstract}

Keywords: (FP-LMTO) Method, $\mathrm{Cu}_{\mathrm{x}} A g_{1-\mathrm{x}}$ I Alloy

\section{Introduction}

Silver iodide and copper iodide have received attention as promising materials for applications in technological devices and are used as model systems in both physical and numerical experiments [1]. These compounds which have four valence electrons per atoms, crystallize in a tetrahedral coordinated zinc-blende (ZB) structure [2,3] under ambient conditions. Silver iodide can exist in three crystal polymorphs at atmospheric pressure. Below 420 $\mathrm{K}$ silver iodide crystallizes in the hexagonal $\beta$ phase (wurtzite structure). Copper iodide also has several transformations. The low-temperature cubic $\gamma$-CuI phase with the zinc blende structure, transforms to the $\beta$ phase with a distorted hexagonal structure and a rock salt structure above $673 \mathrm{~K}(\alpha-\mathrm{CuI})$. Another interesting feature of these systems is that the lattice mismatch between $\mathrm{CuI}$ and $\mathrm{AgI}$ is very small and therefore it is useful to combine them for forming $\mathrm{Cu}_{x} \mathrm{Ag}_{1-x} \mathrm{I}$ alloys. Indeed, $\mathrm{CuI}$ and $\mathrm{AgI}$ binary compounds form a continuous series of alloys $\left(\mathrm{Cu}_{x} \mathrm{Ag}_{1-x} \mathrm{I}\right)$, where $0<x<1$ denotes the molar fraction of $\mathrm{Cu}$. Both $\mathrm{CuI}$ and $\mathrm{AgI}$ crystallizes in the cubic zinc blende structure. Hence, one would expect that the different properties of the ternary alloy to be vary smooth- ly between the end points. Moreover, it was shown experimentally [4] that the $\alpha-\mathrm{Cu}_{x} \mathrm{Ag}_{1-x} \mathrm{I}$ based solid solutions in the quasibinary $\mathrm{AgI}-\mathrm{CuI}$ system exists over a wide temperature range. The ionic transport in $\mathrm{Cu}_{x} \mathrm{Ag}_{1-x} \mathrm{I}$ was studied by Nölting and co-workers and Kusakabe et al. $[4,5]$. They have observed that the electric conductivity reduced with increasing the copper concentration.

Goldmann [6] reviewed detailed experimental and theoretical information of the valence and conduction band states in CuI, and AgI. He proposed a band structure model which is able to interpret all available experimental information extracted from optical absorption spectroscopy, soft X-ray absorption and emission spectroscopy. Theoretically, many calculations using the molecular dynamics simulations $[7,8]$ have been done to characterize the structural, electronic, and optical properties of these systems.

Thus our main goal of this work is to study the effect of increasing concentration of copper on the structural and electronic properties band structure such as lattice constant, bulk modulus, band gaps and effective masses by using the full potential linear muffin-tin orbitals (FP-LMTO) method. In this work, we have used the "special quasirandom structures" (SQS) approach of 
Zunger et al. [9] to reproduce the randomness of the alloys for the first few shells around a given site. This approach is reasonably sufficient to describe the alloys with respect to many physical properties that are not affected by the errors introduced using the concept of the periodicity beyond the first few shells.

The organization of the article is as follows. The computational method we have adopted for the calculations is described in Section 2. We present our results in Section 3. Finally, conclusions are given in Section 4.

\section{Method of Calculations}

The calculations reported here were carried out using the ab-initio full-potential linear muffin-tin orbital (FPLMTO) method [10-13] as implemented in the Lmtart code [14]. The exchange and correlation potential was calculated using the local density approximation (LDA) [15] and the generalized approximation (GGA) [16]. This is an improved method compared to previous (LMTO) methods. The FP-LMTO method treats muffin-tin spheres and interstitial regions on the same footing, leading to improvements in the precision of the eingenvalues. At the same time, the FP-LMTO method, in which the space is divided into an interstitial regions (IR) and non overlapping muffin-tin spheres (MTS) surrounding the atomic sites, uses a more complete basis than its predecessors. In the IR regions, the basis functions are represented by Fourier series. Inside the MTS spheres, the basis functions are represented in terms of numerical solutions of the radial Schrödinger equation for the spherical part of the potential multiplied by spherical harmonics. The charge density and the potential are represented inside the MTS by spherical harmonics up to $1_{\max }=6$. The integrals over the Brillouin zone are performed up to 42 special $k$-points for binary compounds and 32 special $k$-points for the alloys in the irreducible Brillouin zone (IBZ), using the Blöchl's modified tetrahedron method [17]. The self-consistent calculations are considered to be converged when the total energy of the system is stable within $10^{-5} \mathrm{Ry}$. In order to avoid the overlap of atomic spheres the MTS radius for each atomic position is taken to be different for each case. Both the plane waves cut-off are varied to ensure the total energy convergence. The values of the sphere radii (MTS), number of plane waves (NPLW), used in our calculation are summarized in Table $\mathbf{1 .}$

\section{Results and Discussions}

\subsection{Structural Properties}

As a first step we calculate the structural properties of the compounds CuI, AgI and their alloy in the cubic structure by means of full-potential LMTO method. As for the semiconductor ternary alloys in the type $\mathrm{B}_{x} \mathrm{~A}_{1-x} \mathrm{C}$, we have started our FP-LMTO calculation of the structural properties with the zinc-blende structure and let the calculation forces to move the atoms to their equilibrium positions. We have chosen the basic cubic cell as the unit cell. In the unit cell there are four $\mathrm{C}$ anions and three $\mathrm{A}$ and one $\mathrm{B}$, two $\mathrm{A}$ and two $\mathrm{B}$, and one $\mathrm{A}$ and three $\mathrm{B}$ cations, respectively, for $x=0.25,0.50$ and 0.75 . For the considered structures, we perform the structural optimization by calculating the total energies for different volumes around the equilibrium cell volume $\mathrm{V}_{0}$ for $\mathrm{CuI}$ and AgI binary compounds and their alloys. The calculated total energies are fitted to the Murnaghan's equation of state [18] to determine the ground state properties such as the equilibrium lattice constant $a$, and the bulk modulus

Table 1. The plane wave number PW, energy cut-off (in Ry) and the muffin-tin radius (RMT) ( in a.u.) used in calculation for binary $\mathrm{AgI}$ and $\mathrm{CuI}$ and their alloy in zinc blende (ZB) structure.

\begin{tabular}{|c|c|c|c|c|c|c|c|}
\hline \multirow[b]{2}{*}{$x$} & \multicolumn{2}{|c|}{ PW } & \multicolumn{2}{|c|}{$\mathrm{E}_{\text {cut }}$ total (Ry) } & \multicolumn{3}{|c|}{ RMT (a.u) } \\
\hline & LDA & GGA & LDA & GGA & & LDA & GGA \\
\hline \multirow[t]{2}{*}{0} & 5064 & 12050 & 75.918 & 123.177 & $\mathrm{Ag}$ & 2.490 & 2.607 \\
\hline & & & & & I & 2.808 & 2.939 \\
\hline \multirow[t]{3}{*}{0.25} & 33400 & 65266 & 108.971 & 155.912 & $\mathrm{Cu}$ & 2.449 & 2.560 \\
\hline & & & & & $\mathrm{Ag}$ & 2.449 & 2.560 \\
\hline & & & & & I & 2.762 & 2.845 \\
\hline \multirow[t]{3}{*}{0.50} & 33400 & 65266 & 113.796 & 163.276 & $\mathrm{Cu}$ & 2.350 & 2.477 \\
\hline & & & & & $\mathrm{Ag}$ & 2.350 & 2.477 \\
\hline & & & & & I & 2.750 & 2.845 \\
\hline \multirow[t]{3}{*}{0.75} & 33400 & 65266 & 118.805 & 171.247 & $\mathrm{Cu}$ & 2.300 & 2.418 \\
\hline & & & & & $\mathrm{Ag}$ & 2.300 & 2.418 \\
\hline & & & & & I & 2.691 & 2.778 \\
\hline \multirow[t]{2}{*}{1} & 5064 & 9984 & 90.1556 & 131.503 & $\mathrm{Cu}$ & 2.180 & 2.267 \\
\hline & & & & & I & 2.674 & 2.771 \\
\hline
\end{tabular}


$B$. The calculated equilibrium parameters $(a$ and $B$ ) are given in Table 2 which also contains results of previous calculations as well as the experimental data. On the whole, our calculated structural parameters are in good agreement with those obtained by first-principles methods within different approximations. The calculated lattice parameters values for the binary compounds are in good agreement with the measured data, which ensure the reliability of the present first-principles computations. The computed lattice parameters slightly underestimates and overestimates the measured data by using LDA and GGA, respectively, which are consistent with the general trend of these approximations. As it can be seen that the calculated lattice parameter for $\mathrm{CuI}(\mathrm{x}=1)$ is smaller than those of $\mathrm{AgI}(\mathrm{x}=0) ; a_{0}(\mathrm{CuI})<a_{0}(\mathrm{AgI})$. As the anion atom is the same in both compounds, this result can be easily explained by considering the atomic radii of $\mathrm{Cu}$ and $\mathrm{Ag}: R(\mathrm{Cu})=1.35 \AA, R(\mathrm{Ag})=1.60 \AA$, i.e., the lattice constant increases with increasing atomic size of the cation element. The bulk modulus value for $\mathrm{CuI}$ is larger than those of AgI; B $(\mathrm{CuI})>B(\mathrm{AgI})$; i.e., in inverse sequence to $a_{0}$-in agreement with the well-known relationship between $B$ and the lattice constants: $B \propto V_{0}^{-1}$, where $V_{0}$ is the unit cell volume. Furthermore, the values of the calculated bulk modulus using both approximations decreases from $\mathrm{CuI}$ to $\mathrm{AgI}$, suggesting that the compressibility increases from $\mathrm{CuI}$ to $\mathrm{AgI}$.

Usually, in the treatment of alloys when the experimental data are scare, it is assumed that the atoms are located at the ideal lattice sites and the lattice constant varies linearly with composition $x$ according to the socalled Vegard's law [19].

$$
a\left(A_{x} B_{1-x} C\right)=x a_{A C}+(1-x) a_{B C}
$$

where $a_{A C}$ and $a_{B C}$ are the equilibrium lattice constants of the binary compounds $\mathrm{AC}$ and $\mathrm{BC}$ respectively, $a\left(A_{x} B_{1-x} C\right)$ is the alloy lattice constant. However, vio- lation of Vegard's law has been observed in semiconductor alloys both experimentally [20] and theoretically [21].

Hence, the lattice constant can be written as:

$$
a\left(A_{x} B_{1-x} C\right)=x a_{A C}+(1-x) a_{B C}-x(1-x) b
$$

where the quadratic term $b$ is the bowing parameter.

Figures 1 and 2, show the variation of the calculated equilibrium lattice constants and the bulk modulus versus concentration $x$ for $\mathrm{Cu}_{x} \mathrm{Ag}_{1-x} \mathrm{I}$ alloy. The obtained results for the composition dependence of the calculated equilibrium lattice parameter show an excellent agreement to Vegard's law [19]. In going from AgI to CuI; when the $\mathrm{Cu}$-content increases, the values of the lattice parameters of the $\mathrm{Cu}_{x} \mathrm{Ag}_{1-x} \mathrm{I}$ alloy decrease. This is due to the fact that the size of the $\mathrm{Cu}$ atom is smaller than those of $\mathrm{Ag}$ atom. Oppositely, one can see from Figure 2 that the value of the bulk modulus increases with increasing $\mathrm{Cu}$ concentration. The values of the bowing parameters are determined by a polynomial fit. Using the LDA approximation, we obtained upward and downward bowing parameters equal to $-0.11543 \AA$ and $0.01143 \mathrm{GPa}$ for the lattice and the bulk modulus, respectively. The GGA approximation gave values of $-0.11543 \AA$ and 13.95429 GPa for the bowing lattice parameter and the bulk modulus, respectively. In view of Table 2, it is clear that both approximations follow the tendency demonstrated by both experimental measurement and theoretical calculations. To the best of our knowledge, there are no experimental work exploring the structural properties (e.g.; the bulk modulus B and it pressure derivatives $\mathrm{B}^{\prime}$ ) and the bowing parameters of the investigated alloys, but our results are relatively close to those of Ref. [22] obtained by using the full potential-linear augmented plane wave (FP-LAPW) method.

\subsection{Electronic Properties}

The energy band gaps of the binary compounds as well

Table 2. Calculated lattice parameter $a$ and bulk modulus $B$ compared to experimental and other theoretical values of $\mathrm{CuI}$

\begin{tabular}{|c|c|c|c|c|c|c|c|c|}
\hline \multicolumn{6}{|c|}{ Lattice constant a $(\AA ̊)$} & \multicolumn{3}{|c|}{ Bulk modulus $B(\mathrm{Gpa})$} \\
\hline & \multicolumn{2}{|c|}{ this work. } & \multirow[t]{2}{*}{ expt. } & \multirow[t]{2}{*}{ other calc. } & \multicolumn{2}{|c|}{ this work. } & \multirow[t]{2}{*}{ expt. } & \multirow[t]{2}{*}{ other calc. } \\
\hline & LDA & GGA & & & LDA & GGA & & \\
\hline \multicolumn{9}{|l|}{$x$} \\
\hline 0 & 6.476 & 6.779 & $6.499^{\mathrm{a}}$ & $6.650^{\mathrm{c}}, 6.61^{\mathrm{d}}, 6.872^{\mathrm{e}}$ & 34.26 & 23.82 & $24^{\mathrm{j}}$ & $26.667^{\mathrm{c}}, 40^{\mathrm{l}}$ \\
\hline 0.25 & 6.37 & 6.646 & & $6.516^{\mathrm{c}}, \quad 6,39^{\mathrm{f}}, 6.38^{\mathrm{g}}$ & 37.93 & 24.7 & & $27.405^{\mathrm{c}}, 20,3^{\mathrm{f}}, 26.0^{\mathrm{g}}$ \\
\hline 0.5 & 6.234 & 6.505 & & $6.396^{\mathrm{c}}, 6.31^{\mathrm{f}}, 6.30^{\mathrm{g}}$ & 42.2 & 26.61 & & $30,104^{\mathrm{c}}, 23^{\mathrm{f}}, 27^{\mathrm{g}}$ \\
\hline 0.75 & 6.101 & 6.352 & & $6.256^{\mathrm{c}}, 6.19^{\mathrm{f}}, 6.18^{\mathrm{g}}$ & 45.58 & 29.55 & & $37.765^{\mathrm{c}}, 27^{\mathrm{f}}, 28^{\mathrm{g}}$ \\
\hline 1 & 5.943 & 6.175 & $6.054^{\mathrm{b}}$ & $6.098^{\mathrm{c}}, 6.097^{\mathrm{h}}, 6.082^{\mathrm{i}}$ & 49.7 & 36.02 & $36.6^{\mathrm{b}}, 31^{\mathrm{k}}$ & $38.872^{\mathrm{c}}, 39.447^{\mathrm{h}}, 39.7^{\mathrm{i}}, 35.2^{\mathrm{m}}$ \\
\hline
\end{tabular}
and AgI and their alloys.

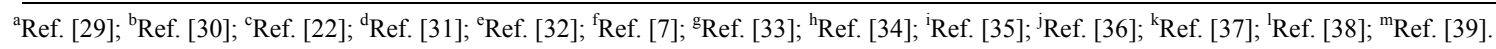




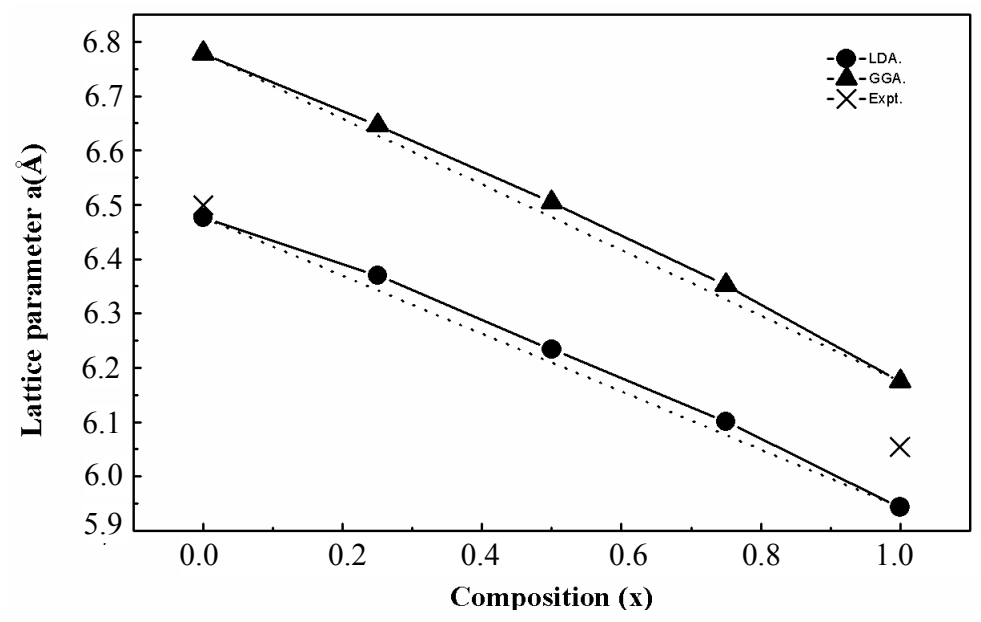

Figure 1. Composition dependence of the calculated lattice constants within LDA (Solid Circle) and GGA (Solid Triangle) of $\mathrm{Cu}_{x} \mathbf{A g}_{1-x} \mathrm{I}$ alloy compared with Vegard's prediction (doted line).

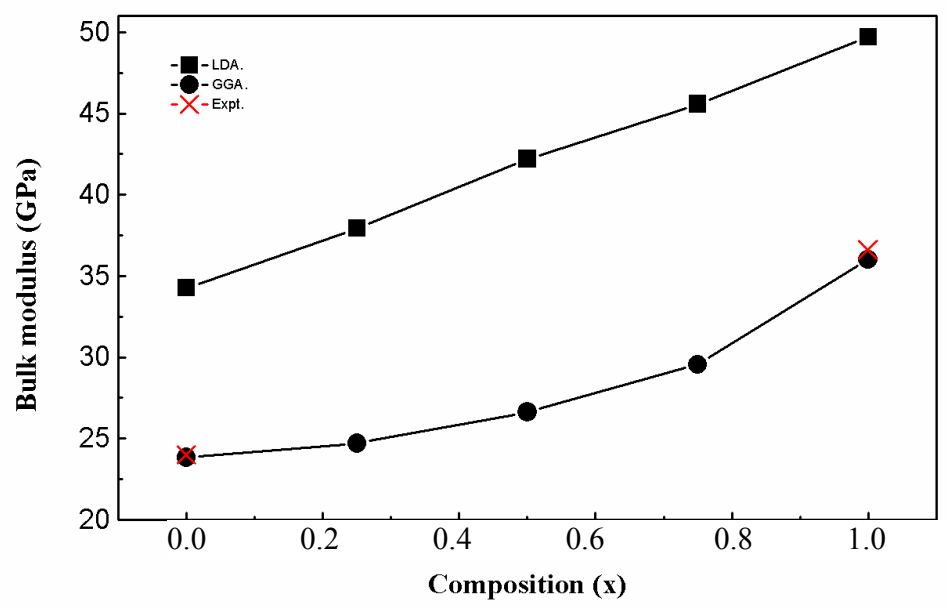

Figure 2. Composition dependence of the calculated bulk modulus within GGA (Solid Circle) and LDA (Solid Square) of $\mathrm{Cu}_{x} \mathrm{Ag}_{1-x}$ I alloy.

as for their investigated alloy were calculated within the LDA and GGA schemes. In Table 3 we show the obtained results for the energy band gap of the $\mathrm{Cu}_{x} \mathrm{Ag}_{1<-x} \mathrm{I}$ alloy from our ab initio calculations for different values of the concentration $x$. These values correspond to 25, 50, 75 and $100 \% \mathrm{CuI}$ and $\mathrm{AgI}$ substitution, respectively. The exact value of the band-gap is obviously a crucial point to be addressed because it enters in the applications in technological devices. However, it can be seen from Table 3 that there is a large discrepancy between the reported experimental and theoretical values. This discrepancy is mainly due to the fact that both the simple form of LDA or GGA do note take into account the quasiparticle self energy correctly [23] which make them not sufficiently flexible to accurately reproduce both exchange and correlation energy and its charge derivative. We worth also mention that in general, it is far to say that the experimental data are well reproduced by the calculation. On raison for this difference is that in our calculations we have assumed the crystal to be at $T=$ $0 \mathrm{~K}$ and thus do not include contributions from lattice vibrations that are present at room temperature measurements. It is important to note that the density functional formalism is limited in its validity (see. Ref [24]) and the band structure derived from it cannot be used directly for comparison with experiment. The variation of the concentration $(x)$ versus the value of the direct band gap energy $\left(^{\sim}\right.$. is with both LDA and GGA approximation is shown in Figure 3. It is clearly seen that the direct energy gap show a non linear variation with copper concentration. The obtained energy versus concentration curve is well fitted by the following quadratic phenomenological function. 
Table 3. Calculated direct energy band gap energy $(\Gamma-\Gamma)$ of $\mathrm{Cu}_{x} \mathbf{A g}_{1-x} I$ alloys at different $\mathrm{Cu}$ concentrations (all values are in eV).

\begin{tabular}{|c|c|c|c|c|}
\hline \multirow[b]{3}{*}{$x$} & \multicolumn{4}{|c|}{$\mathrm{E}_{\mathrm{g}}(\mathrm{eV})$} \\
\hline & \multicolumn{2}{|c|}{ this work. } & \multirow[t]{2}{*}{ expt } & \multirow[t]{2}{*}{ other calc. } \\
\hline & LDA & GGA & & \\
\hline 0 & 1.171 & 1.416 & & $1.340^{\mathrm{c}}, 2.077^{\mathrm{c}}, 1.4^{\mathrm{b}}, 0.8^{\mathrm{d}}$ \\
\hline 0.25 & 1.012 & 1.106 & & $1.04632^{\mathrm{c}}, 1.68286^{\mathrm{c}}$ \\
\hline 0.5 & 0.979 & 0.993 & & $0.958^{\mathrm{c}}, 1.59617^{\mathrm{c}}$ \\
\hline 0.75 & 1.020 & 0.962 & & $0.965^{\mathrm{c}}, 1.61436^{\mathrm{c}}$ \\
\hline 1 & 1.183 & 1.018 & $2.95^{\mathrm{a}}$ & $1.068^{\mathrm{c}}, 1.764^{\mathrm{c}}, 1.077^{\mathrm{e}}, 1.118^{\mathrm{f}}, 3.10^{\mathrm{g}}$ \\
\hline
\end{tabular}

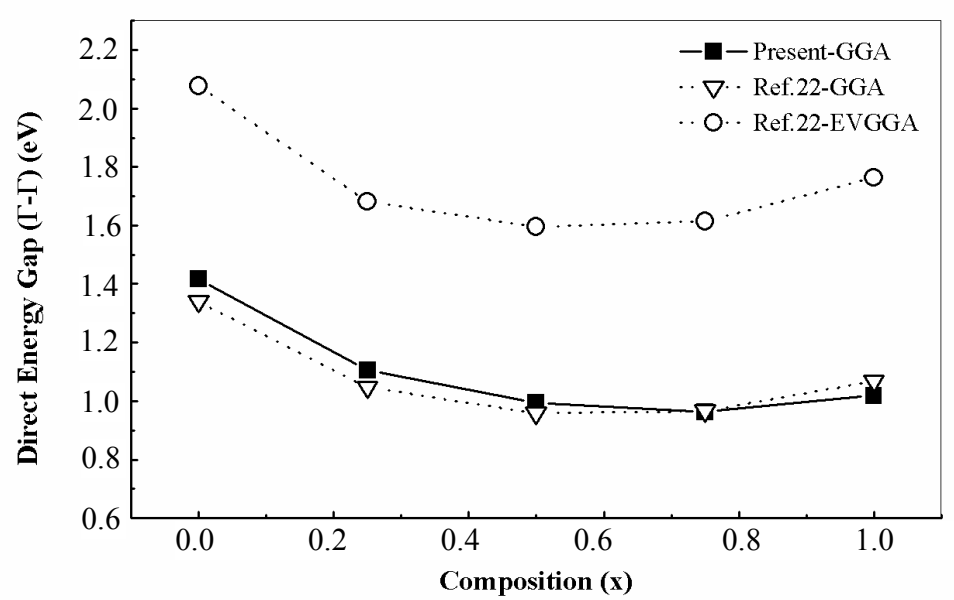

Figure 3. Calculated direct energy band gap $(\Gamma-\Gamma)$ of $\mathrm{Cu}_{x} \mathrm{Ag}_{1-x} I$ alloys as a function of Cu concentration using GGA approximation (solid square-solid line) compared with Ref. 22, using GGA (Open Triangle-doted line) and EVGGA (Open Circle-doted line).

$$
E_{g}(x)=x E_{A C}+(1-x) E_{B C}-b x(1-x)
$$

where $E_{A C}$ and $E_{B C}$ corresponds to the gap of the $\mathrm{CuI}$ and $\mathrm{AgI}$ for the $\mathrm{Cu}_{x} \mathrm{Ag}_{1-X} \mathrm{I}$ alloy.

The obtained results are shown in Figure $\mathbf{3}$ and are summarized as follows:

$$
\mathrm{Cu}_{x} \operatorname{Ag}_{1-x} \mathrm{I} \rightarrow \begin{cases}\mathrm{E}_{\Gamma-\Gamma}=1.67-0.81 x+0.82 x^{2} & (L D A) \\ \mathrm{E}_{\Gamma-\Gamma}=1.40-1.31 x-0.93 x^{2} & (G G A)\end{cases}
$$

In order to better understand the physical origins of bowing parameters in $\mathrm{A}_{x} \mathrm{~B}_{1-x} \mathrm{C}$ alloys, we follow the procedure of Bernard and Zunger [25], and decompose the total bowing parameter $b$ into physically distinct contributions. The overall bowing coefficient at a given average composition $x$ measures the change in the band gap according to the formal reaction

$$
x \mathrm{AC}\left(a_{A C}\right)+(1-x) \mathrm{BC}\left(a_{B C}\right) \rightarrow A_{x} B_{1-x} C\left(a_{e q}\right)
$$

where $a_{A C}$ and $a_{B C}$ are the equilibrium lattice constants of the binary compounds and $a_{e q}$ is the equilib- rium lattice constant of the alloy with the average composition $x$.

The Equation (5) is decomposed into three steps:

$$
\begin{gathered}
\mathrm{AC}\left(a_{A C}\right)+\mathrm{BC}\left(a_{B C}\right) \rightarrow \mathrm{AC}(a)+B C(a) \\
x \mathrm{AC}(a)+(1-x) \mathrm{BC}(a) \rightarrow A_{x} B_{1-x} C(a) \\
A_{x} B_{1-x} C(a) \rightarrow A_{x} B_{1-x} C\left(a_{e q}\right)
\end{gathered}
$$

The first step measures the volume deformation (VD) effect on the bowing. The corresponding contributions $b_{\mathrm{VD}}$ to the bowing parameter represents the relative response of the band structure of the binary compounds AC and $\mathrm{BC}$ to hydrostatic pressure, which here arises from the change of their individual equilibrium lattice constants to the alloy value $a=a(x)$. The second contribution, the charge exchange $(\mathrm{CE})$ contribution $b_{\mathrm{CE}}$, reflects the charge transfer effect which is due to the different (averaged) bonding behavior at the lattice constant $a$. The final step measure changes due to the structural relaxation (SR) in passing from the unrelaxed to the relaxed alloy by $b_{\mathrm{SR}}$. Consequently, the total bowing parameter is de- 
fined as

$$
b=b_{\mathrm{VD}}+b_{\mathrm{CE}}+b_{\mathrm{SR}}
$$

The general representation of the composition-dependent band gap of the alloys in terms of binary compounds gaps of the, $E_{A C}\left(a_{A C}\right)$ and $E_{B C}\left(a_{B C}\right)$, and the total band gap bowing parameter $b$ is defined as:

$$
E_{g}(x)=x E_{A C}\left(a_{A C}\right)+(1-x) E_{B C}\left(a_{B C}\right)-b x(1-x)
$$

This allows a division of the total gap bowing $b$ into three contributions according the following expressions:

$$
\begin{gathered}
b_{V D}=\frac{E_{A C}\left(a_{A C}\right)-E_{A C}(a)}{1-x}+\frac{E_{B C}\left(a_{B C}\right)-E_{B C}(a)}{x} \\
b_{C E}=\frac{E_{A C}(a)}{1-x}+\frac{E_{B C}(a)}{x}-\frac{E_{A B C}(a)}{x(1-x)}
\end{gathered}
$$

$$
b_{S R}=\frac{E_{A B C}(a)-E_{A B C}\left(a_{e q}\right)}{x(1-x)}
$$

All these energy gaps occurring in expressions (11)(13) have been calculated for the indicated atomic structures and lattice constants. Table 4 shows our results for the optical band gap bowing $b$, as calculated for three different molar fractions $(x=0.25,0.5$ and 0.75$)$. The calculated GGA-band gap bowing parameter exhibits strong composition dependence. This is different from LDA calculations, which show a weakly composition dependent bowing parameter. Indeed, Figure 4, shows the variation of the band gap bowing versus concentration. The bowing remains linear and varies slowly in

\begin{tabular}{|c|c|c|c|c|c|}
\hline & & \multicolumn{2}{|c|}{ this work. } & \multicolumn{2}{|c|}{ other calc. } \\
\hline & & LDA & GGA & & \\
\hline \multicolumn{6}{|l|}{$x$} \\
\hline \multirow[t]{4}{*}{0.25} & $b_{\mathrm{VD}}$ & 0.5896 & 0.5435 & & \\
\hline & $\mathrm{b}_{\mathrm{CE}}$ & 0.7138 & 0.7338 & & \\
\hline & $b_{\mathrm{SR}}$ & -0.439 & 0.0498 & & \\
\hline & $\mathrm{b}$ & 0.8629 & 1.3273 & & \\
\hline \multirow[t]{4}{*}{0.5} & $b_{\mathrm{VD}}$ & 0.5964 & 0.5963 & $0.55252^{\mathrm{a}}$ & $0.5719^{\mathrm{a}}$ \\
\hline & $\mathrm{b}_{\mathrm{CE}}$ & 0.1270 & 0.2490 & $0.9184^{\mathrm{a}}$ & $1.21098^{\mathrm{a}}$ \\
\hline & $\mathrm{b}_{\mathrm{SR}}$ & 0.0629 & 0.0585 & $0.06772^{\mathrm{a}}$ & $0.08624^{\mathrm{a}}$ \\
\hline & $\mathrm{b}$ & 0.7863 & 0.8939 & $1.53864^{\mathrm{a}}$ & $1.86912^{\mathrm{a}}$ \\
\hline \multirow[t]{4}{*}{0.75} & $b_{\mathrm{VD}}$ & 0.6408 & 0.6029 & & \\
\hline & $\mathrm{b}_{\mathrm{CE}}$ & 0.0789 & 0.1033 & & \\
\hline & $b_{\mathrm{SR}}$ & 0.1315 & 0.1250 & & \\
\hline & $\mathrm{b}$ & 0.8512 & 0.8313 & & \\
\hline
\end{tabular}
going from $x=0.0$ to $x=1.0$. The calculated gap bowing coefficient for random $\mathrm{Cu}_{x} \mathrm{Ag}_{1-x} \mathrm{I}$ alloy ranges from

Table 4. Decomposition of optical bowing into volume deformation (VD), charge exchange (CE) and structural relaxation (SR) contributions (all values in $\mathrm{eV}$ ).

${ }^{\mathrm{a}}$ Ref. [22]

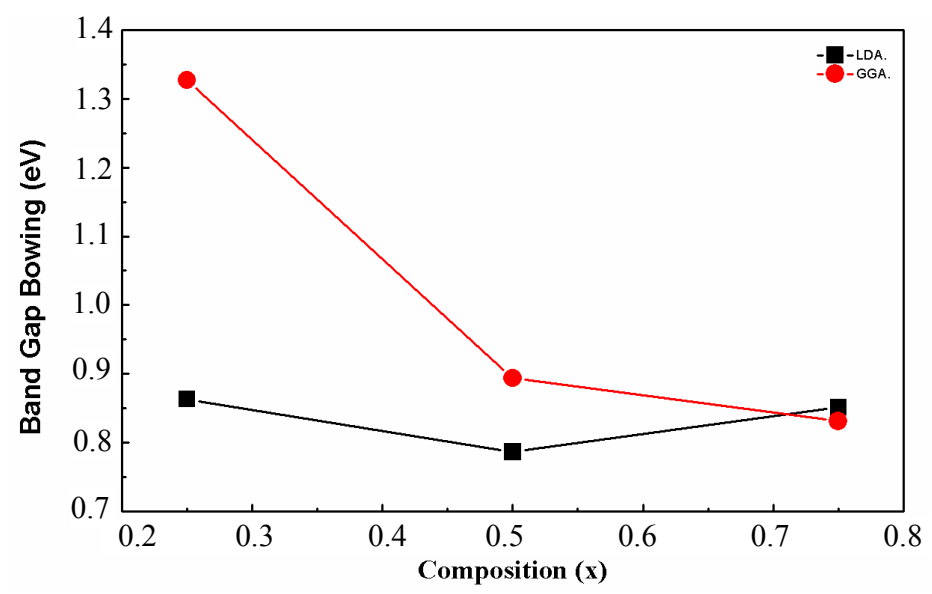

Figure 4. Calculated band gap bowing parameter as a function of Cu concentration with LDA (Solid Square) and GGA ( Solid Circle). 
$0.8629 \mathrm{eV}(x=0.25)$ to $0.8512(x=0.75)$. Our result for $x=0.5$ is in excellent agreement with those obtained by using EVGGA-FPLAPW method [22]. We can also note that the volume-deformation term $b_{V D}$ of $\mathrm{Cu}_{x} \mathrm{Ag}_{1-x} \mathrm{I}$ is large. The importance of $b_{V D}$ can be correlated with the mismatch of the lattice constants of the corresponding binary compounds. As consequence, the main contribution to the gap bowing is raised from the volume deformation effect. The only exception is for $x=0.25$ in which the charge transfer contribution $b_{C E}$ has been found greater than $b_{V D}$. This contribution is due to the different electronegativities of the $\mathrm{Ag}$ and $\mathrm{Cu}$ or I atoms. Indeed, $b_{C E}$ scales with the electronegativity mismatch. The contribution of structural relaxation is negligible; the band gap bowing is due essentially to the charge exchange effect. We conclude that our calculations show that both structural (volume deformation) and chemical effects appear to control the optical gap bowing of $\mathrm{Cu}_{x} \mathrm{Ag}_{1-\chi} \mathrm{I}$ alloy.

\subsection{Calculated Effective Masses}

Knowledge of the electron and hole effective mass values is indispensable for the understanding of transport phenomena, exciton effects and electro-hole in semiconductors. Excitonic properties are of great interest for binary $\mathrm{CuI}, \mathrm{AgI}$ and their alloys, therefore, it is worthwhile to estimate the electron and hole effective mass values for these materials. Experimentally, the effective masses are usually determined by cyclotron resonance, electro reflectance measurements or from analysis of transport data or transport measurements [43]. Theoretically, the effective masses can be estimated from the energy band curvatures. Generally, the effective mass is a tensor with nine components, however for the much idealized simple case, where the $E-k$ diagram can be fitted by a parabola $E=\hbar^{2} k^{2} / 2 m^{*}$, the effective mass becomes a scalar at high symmetry point in Brillouin zone. We have computed the electron effective mass at the conduction band minima (CBM) and the hole effective mass at the valence band maxima (VBM) for CuI, AgI and their ternary alloys. The electron and the hole effective masses are obtained from the curvature of the energy band near the $\Gamma$ point at the conduction band minimum (CBM) and valence band maximum (VBM), respectively. The calculated electron and hole effective masses values for the parent binary compounds $\mathrm{CuI}$ and $\mathrm{AgI}$ and their alloy are given in Table 5. Results from earlier theoretical works are also quoted for comparison. The calculated electron effective masses are in good agreement with those obtained by using the full potential linear augmented plane wave (FP-LAPW) method whin EVGGA approximation [22] which is known to improve the values of the band gaps rather than LDA and GGA [26-28]. This agreement disappears in the case of hole effective masses. This disagreement could be expected because the computation of the effective mass is very sensitive to the topology of band structure. The highest curvature of the electronic band yields the smallest effective mass of the charge carriers and the highest conductivity. Therefore, the effective masses that depend strongly on the form of energy band match the expected accuracy. From Table 5 data, we can outline that holes are much heavier than electrons, for all concentrations in $\mathrm{Cu}_{x} \mathrm{Ag}_{1-x} \mathrm{I}$ alloy, so carrier transport in this alloy should be dominated by electrons. We notice that no experimental data are available for effective masses of this alloy; all results are obtained via theoretical methods. Future experimental work will testify all calculated results.

\section{Conclusions}

In summary, by means of the (FP-LMTO) method, we have calculated the structural and the electronic properties of zinc-blende $\mathrm{CuI}, \mathrm{AgI}$, and their related $\mathrm{Cu}_{x} \mathrm{Ag}_{1-x} \mathrm{I}$ alloy. We have found that except the lattice parameter, the variation of structural parameters versus copper concentration not obeys Vegard's law. Our results of the electronic band structure show a non linear variation of the fundamental ban gaps versus copper concentration.

Table 5. The electron and hole effective masses for $\mathrm{Cu}_{x} \mathrm{Ag}_{1-x} I$ ternary alloy as function of $\mathrm{Cu}$ concentrations using $\mathrm{LDA}$ and GGA. All values are in units of a free-electron mass $\mathbf{m}_{0}$.

\begin{tabular}{|c|c|c|c|c|c|c|c|c|c|c|c|}
\hline & \multicolumn{4}{|c|}{$m_{e}{ }^{*}$} & \multicolumn{3}{|c|}{$m_{h h}{ }^{*}$} & \multicolumn{4}{|c|}{$m_{l h}{ }^{*}$} \\
\hline & \multicolumn{2}{|c|}{ this work. } & \multicolumn{2}{|c|}{ other calc. } & \multicolumn{2}{|c|}{ this work. } & \multirow[t]{2}{*}{ other calc. } & \multicolumn{2}{|c|}{ this work. } & \multicolumn{2}{|c|}{ other calc. } \\
\hline$x$ & LDA & GGA & & & LDA & GGA & & LDA & GGA & & \\
\hline 0 & 0.271 & 0.270 & $0.226^{\mathrm{a}}$ & $0.272^{\mathrm{a}}$ & 0.949 & 0.960 & & 1.818 & 1.804 & $1.187^{\mathrm{a}}$ & $1.189^{\mathrm{a}}$ \\
\hline 0.25 & 0.226 & 0.329 & $0.197^{\mathrm{a}}$ & $0.261^{\mathrm{a}}$ & 0.500 & 0.634 & & 0.524 & 0.642 & $1.355^{\mathrm{a}}$ & $1.622^{\mathrm{a}}$ \\
\hline 0.50 & 0.3006 & 0.306 & $0.191^{\mathrm{a}}$ & $0.256^{\mathrm{a}}$ & 0.439 & 0.577 & & 0.466 & 0.591 & $1.281^{\mathrm{a}}$ & $1.462^{\mathrm{a}}$ \\
\hline 0.75 & 0.238 & 0.285 & $0.188^{a}$ & $0.251^{\mathrm{a}}$ & 0.453 & 0.5339 & & 0.465 & 0.541 & $1.185^{\mathrm{a}}$ & $1.318^{\mathrm{a}}$ \\
\hline 1 & 0.229 & 0.220 & $0.186^{\mathrm{a}}$ & $0.249^{\mathrm{a}}$ & 0.573 & 0.601 & & 0.804 & 0.768 & $1.163^{\mathrm{a}}$ & $1.158^{\mathrm{a}}$ \\
\hline
\end{tabular}

${ }^{\mathrm{a}}$ Ref. [22]. 
We have characterized the deviation from the linear behavior by calculating the optical bowing parameter. The effective masses of the systems studied in this work were calculated and are found comparable to those obtained by FPLAPW method.

\section{Acknowledgements}

Author Rabah KHENATA extends his appreciation to the Deanship of Scientific Research at King Saud University for funding the work through the research group project N0 RPG-VPP-088

\section{REFERENCES}

[1] A. K. Ivanov-Shits and I. V. Murin "Ionika Tverdogo tela," Izd-vo SPbGU, S.-Peterburg, 2000.

[2] J. G. P. Binner, G. Dimitrakis, D. M. Price1, M. Reading and B. Vaidhyanathan, "Hysterisis in the Phase Transition in Silver Iodide," Journal of Thermal Analysis and Calorimetry, Vol. 84, No. 2, 2006, pp. 409-412. doi:10.1007/s10973-005-7154-1

[3] F. Wells, "Structural Inorganic Chemistry," Oxford University Press, Oxford, 1984.

[4] J. Nölting and Ber. Bunsenges, Physik. Chem., Vol. 68. No. 10, 1964, p. 932.

[5] M. Kusakabe, Y. Shirakawa, S. Tamaki and Y. Ito, "The Transport Properties of Cations in the Superionic Phase of $\mathrm{Ag}_{\mathrm{x}} \mathrm{Cu}_{1-\mathrm{x}} \mathrm{I}$," Journal of the Physical Society of Japan, Vol. 64, No. 01, 1995, pp. 170-176.

[6] A. Goldmann, "Band Structure and Optical Properties of Tetrahedrally Coordinated Cu- and Ag-Halides," Physica Status Solidi (B), Vol. 81, No 1, 1977, pp. 9-47.

[7] W. Sekkal, A. Laref and H. Aourag, A. Zaoui and M. Certier, "The miscibility of $\mathrm{Cu}_{\mathrm{x}} \mathrm{Ag}_{1-\mathrm{x}} \mathrm{I}$ Using a Tersoff Potential," Superlattice and Microstructures, Vol. 28, No. 1, 2000, pp. 55-66. doi:10.1006/spmi.1999.0782

[8] B. Jarosław and R. Jarosław, "Cation Diffusion Coefficients in $\mathrm{CuAgI}$ via Molecular Dynamics Simulations," Solid State Ionics, Vol. 157, No. 1-4, 2003, pp. 227-232. doi:10.1016/S0167-2738(02)00214-X

[9] A. Zunger, S.-H. Wei, L. G. Ferreira and J. E. Bernard, "Special Quasirandom Structures," Physical Review Letters, Vol. 65, No. 3, 1990, pp. 353-356. doi:10.1103/PhysRevLett.65.353

[10] S. Savrasov and D. Savrasov, "Full-Potential LinearMuffin-Tin-Orbital Method for Calculating Total Energies and Forces," Physical Review B, Vol. 46, No. 19, 1992, pp. 12181-12195. doi:10.1103/PhysRevB.46.12181

[11] S. Y. Savrasov, "Linear-Response Theory and Lattice Dynamics: A Muffin-Tin-Orbital Approach," Physical Review B, Vol. 54, No. 23, 1996, pp. 16470-16486. doi:10.1103/PhysRevB.54.16470

[12] P. Hohenberg and W. Kohn, "Inhomogeneous Electron Gas,” Physical Review, Vol. 136, No. 3B, 1964, pp. B864 -B871.
[13] W. Kohn and L. J. Sham, "Self-Consistent Equations Including Exchange and Correlation Effects," Physical Review A, Vol. 140 , No. 4A, 1965, pp. A1133- A1138. doi:10.1103/PhysRev.140.A1133

[14] S. Y. Savrasov, "Program LMTART for electronic structure calculations," Zeitschrift für Kristallogr, Vol. 220, 2005, pp. 555-557.

[15] J. P. Perdew and Y. Wang, "Accurate and Simple Analytic Representation of the Electron-Gas Correlation Energy," Physical Review A, Vol. 45, No. 13, 1992, pp. 13244-13249. doi:10.1103/PhysRevB.45.13244

[16] J. P. Perdew, S. Burke and M. Ernzerhof, "Generalized Gradient Approximation Made Simple," Physical Review Letters, Vol. 77, No.18, 1996, pp. 3865-3868. doi:10.1103/PhysRevLett.77.3865

[17] P. Blochl, O. Jepsen and O. K. Andersen, "Improved Tetrahedron Method for Brillouin-Zone Integrations," Physical Review B, Vol. 49, No. 23, 1994, pp. 16223 16233. doi:10.1103/PhysRevB.49.16223

[18] F. D. Murnaghan, "The Compressibility of Media under Extreme Pressures," Proceedings of the National Academy of Sciences USA, Vol. 30, 1944, pp. 244-247. doi:10.1073/pnas.30.9.244

[19] L. Vegard, "Formation of Mixed Crystals by Solid-Phase Contact," Zeitschrift für Phys, Vol. 5, No. 5, 1921, pp. 393-395.

[20] B. Jobst, D. Hommel, U. Lunz, T. Gerharda and G. Landwehr, "E0 Band-Gap Energy and Lattice Constant of Ternary $\mathrm{Zn}_{1-\mathrm{x}} \mathrm{Mg}_{\mathrm{x}} \mathrm{Se}$ as Functions of Composition," Applied Physics Letters, Vol. 69, No. 1, 1996, pp. 97-100. doi:10.1063/1.118132

[21] F. El Haj Hassan and H. Akdarzadeh, "First-Principles Investigation of $\mathrm{BN}_{\mathrm{x}} \mathrm{P}_{1-\mathrm{x}}, \mathrm{BN}_{\mathrm{x}} \mathrm{As}_{1-\mathrm{x}}$ and $\mathrm{BP}_{\mathrm{x}} \mathrm{As}_{1-\mathrm{x}}$ Ternary Alloys," Materials Science and Engineering, Vol. 121, 2005, pp. 171-178.

[22] B. Amrani, F. El Haj Hassan, R. Khenata and H. Akbarzadeh, "Theoretical Study of $\mathrm{Cu}_{\mathrm{x}} \mathrm{Ag}_{1-\mathrm{x}} \mathrm{I}$ Alloys," Journal of Physics and Chemistry of Solids, Vol. 70, No. 7, 2009, pp. 1055-1061. doi:10.1016/j.jpcs.2009.05.003

[23] S. N. Rashkeev and W. R. L. Lambrecht, "Second-Harmonic Generation of I-III-VI2 Chalcopyrite Semiconductors: Effects of Chemical Substitutions," Physical Review $B$, Vol. 63, No. 16, 2001, pp. 165212-165224. doi:10.1103/PhysRevB.63.165212

[24] G. Onida, L. Reining, and A. Rubio, "Electronic Excitations: Density-Functional versus Many-Body Green'sFunction Approaches," Reviews of Modern Physics, Vol. 74, 2002, pp. 601-659. doi:10.1103/RevModPhys.74.601

[25] J. E. Bernard and A. Zunger, "Optical Bowing in Zinc Chalcogenide Semiconductor Alloys," Physical Review B, Vol. 34 , No. 8, 1986, pp. 5992-5995.

doi:10.1103/PhysRevB.34.5992

[26] H. Baaziz, Z. Charifi, F. El Haj Hassan, S. J. Hashemifar, and $H$. Akbarzadeh, "FP-LAPW Investigations of $\mathrm{Zn}_{1-\mathrm{x}} \mathrm{Be}_{\mathrm{x}} \mathrm{S}, \mathrm{Zn}_{1-\mathrm{x}} \mathrm{Be}_{\mathrm{x}} \mathrm{Se}$ and $\mathrm{Zn}_{1-\mathrm{x}} \mathrm{Be}_{\mathrm{x}} \mathrm{Te}$ Ternary Alloys," Physica Status Solidi (B), Vol. 243, No. 6, 2006, pp. 1296-1305. 
[27] A. Mokhtari and H. Akbarzadeh, "Ab Initio Calculations of the Electronic and Structural Properties of Beryllium-, Magnesium- and Calcium-Nitrides," Physica B, Vol. 337, 2003, pp. 122-129. doi:10.1016/S0921-4526(03)00387-9

[28] A. Bouhemadou, R. Khenata, F. Zegrar, M. Sahnoun, H. Baltache and A. H. Reshak, "Ab Initio Study of Structural, Electronic, Elastic and High Pressure Properties of Barium Chalcogenides," Computational Materials Science, Vol. 38, No. 2, 2006, pp. 263-270. doi:10.1016/j.commatsci.2006.03.001

[29] S. Hull and D. A. Keen, "Pressure-Induced Phase Transitions in $\mathrm{AgCl}, \mathrm{AgBr}$, and $\mathrm{AgI}$," Physical Review B, Vol. 59, No. 2, 1999, pp. 750-761. doi:10.1103/PhysRevB.59.750

[30] S. Hull and D.A. Keen, "High-Pressure Polymorphism of the Copper(I) Halides: A Neutron-Diffraction Study to 10 GPa," Physical Review B, Vol. 50, No. 9, 1994, pp. 5868-5885. doi:10.1103/PhysRevB.50.5868

[31] L.A. Palomino-Rojas, M. Lo'pez-Fuentes, G. H. Cocoletzi, G. Murrieta, R. de Coss and N. Takeuchi, "Density Functional Study of the Structural Properties of Silver Halides: LDA vs GGA Calculations," Solid State Sciences, Vol. 10, No. 9, pp. 1228-1235. doi:10.1016/j.solidstatesciences.2007.11.022

[32] T. Söhnel, H. Hermann and P. Schwerdtfeger, "Solid State Density Functional Calculations for the Group 11 Monohalides," Journal of Physical Chemistry B, Vol. 109, No. 1, 2005, pp. 526-531.

[33] T. Ida, M. Mizuno, K. Endo, M. Suhara, K. Nishidate and K. Nishikawa, "Dynamical Structure of Alpha-Ag_\{0.99\} $\mathrm{Cu}\{0.01\}$ I Crystal by $\wedge\{63\} \mathrm{Cu}$ NMR Chemical Shift, Spin-Lattice Relaxation Time and Molecular Dyanmics Simulation," Journal of Physics and Chemistry of Solids, Vol. 63, 2002, pp. 249-256. doi:10.1016/S0022-3697(01)00137-8

[34] B. Amrani, T. Benmessabih, M. Tahiri, I. Chiboub, S. Hiadsi and F. Hamdache, "First Principles Study of Structural, Elastic, Electronic and Optical Properties of $\mathrm{CuCl}, \mathrm{CuBr}$ and $\mathrm{CuI}$ Compounds under Hydrostatic Pressure," Physica B, Vol. 381, 2006, pp. 179-186. doi:10.1016/j.physb.2006.01.447
[35] F. El Haj Hassan, A. Zaoui and W. Sekkal, "Structural Properties of Copper Halides," Materials Science and Engineering: B, Vol. 87, 2001, pp. 40-47. doi:10.1016/S0921-5107(01)00687-0

[36] R. C. Hanson, T. A. Fjeldly and H. D. Hochheimer, "Raman Scattering from Five Phases of Silver Iodide," Physica Status Solidi (A), Vol. 70, No. 2, 1975, pp. 567-576. doi: $10.1002 /$ pssb.2220700216

[37] M. Hofmann, S. Hull and D. A. Keen, "High-Pressure Phase of Copper(I) Iodide," Physical Review B, Vol. 51, No. 17, 1995, pp. 12022-12025. doi:10.1103/PhysRevB.51.12022

[38] G. S. Nunes, P. B. Allen and J. L. Martin, "Pressure Induced Phase Transitions in Silver Halides," Physical Review B, Vol. 57, No. 9, 1998, pp. 5098-5105. doi:10.1103/PhysRevB.57.5098

[39] W. Sekkal, A. Zaoui, A. Laref, M. Certier and H. Aourag, "Molecular Dynamics Simulation of CuI using a ThreeBody Potential," Journal of Physics: Condensed Matter, Vol. 12, No. 28, 2000, p. 6173. doi: 10.1088/0953-8984/12/28/313

[40] R. H. Victora, "Calculated Electronic Structure of Silver Halide Crystals," Physical Review B, Vol. 56, No. 8, 1997 , pp. 4417-4421.

[41] V. P. Krüger and J. Pollmann, "Ab Initio Electronic Structure of Silver Halides Calculated with Self-Interaction and Relaxation-Corrected Pseudopotentials," Physical Review B, Vol. 58, No. 7, 1998, pp. 3865-3869. doi:10.1103/PhysRevB.58.3865

[42] M. Ferhat, A. Zaoui, M. Certier, J. P. Dufour and B. Khelifa, "Electronic Structure of the Copper Halides $\mathrm{CuCl}$, $\mathrm{CuBr}$ and Cul," Materials Science and Engineering, Vol. 39, 1996, pp. 95-100. doi:10.1016/0921-5107(95)01518-3

[43] O. Zakharov, A. Rubio, X. Blase, M. L. Cohen and S. G. Louie, "Quasiparticle Band Structures of Six II-VI Compounds: ZnS, ZnSe, ZnTe, CdS, CdSe, and CdTe," Physical Review B, Vol. 50, No. 15, 1994, pp. 10780-10787. doi:10.1103/PhysRevB.50.10780 